\title{
Escalamiento de Procesos Químicos y Bioquímicos basado en un Modelo Fenomenológico
}

\author{
Ángela A. Ruiz ${ }^{(1)}$ y Hernán Álvarez ${ }^{(2)}$ \\ Escuela de Procesos y Energía, Facultad de Minas, Universidad Nacional de Colombia-Medellín. \\ (1) Grupo de Investigación en Bioprocesos y Flujos Reactivos; (2) Grupo de Investigación en \\ Procesos Dinámicos-KALMAN, Cra. 80 x Cl. 65, Barrio Robledo. Medellín -Colombia \\ (e-mail:aaruiz@bt.unal.edu.co)
}

Recibido Dic. 03, 2010; Aceptado Ene. 24, 2011; Versión Final recibida Mar. 27, 2011

\begin{abstract}
Resumen
En este trabajo se presenta un método para el escalamiento de procesos químicos y bioquímicos basada en un modelo de base fenomenológica del proceso. Se definen los conceptos de punto de operación y régimen de operación de un proceso para proponer una herramienta basada en la teoría de control en el escalamiento de procesos, la matriz de Hankel. La metodología se aplicó a un fermentador para la producción de etanol a partir de un jarabe glucosado. Los valores para los parámetros de diseño dan errores inferiores al $10 \%$ al compararlos con los valores de la literatura, tanto para el escalamiento en el laboratorio como para un caso a escala industrial.
\end{abstract}

Palabras clave: escalamiento, bioprocesos, modelo fenomenológico, teoría de control, matriz de Hankel

\section{Scaling of Chemical and Biochemical Processes based on a Phenomenological Model}

\begin{abstract}
In this work a method for the scaling of chemical and biochemical processes based on a phenomenological model is presented. Concepts such as operating point and operating regime of a process are defined to propose a tool based on control theory to process scaling, the Hankel matrix. This methodology was applied to a fermenter for producing ethanol from glucose syrup. The values of the design parameters gave errors below $10 \%$ when they are compared with values reported in the literature, for both laboratory scaling and for an industrial scale.
\end{abstract}

Keywords: scaling, bioprocesses, phenomenological model, control theory, Hankel matrix 


\section{INTRODUCCIÓN}

El escalado de procesos es una de las tareas más importantes tanto durante el diseño de la planta como durante su operación normal. En el primer caso, algunos resultados de producción sólo se conocen a pequeña escala, pero se debe diseñar el proceso a gran escala para que la producción sea rentable. En el segundo caso, ya la planta está construida, pero se deben escalar las condiciones de operación de un nuevo producto que ha sido probado con éxito a pequeña escala, pero que para que sea rentable debe producirse en mayores cantidades. Tradicionalmente se ha realizado el escalado de procesos basándose en la perspectiva del análisis dimensional, en la semejanza geométrica, en las relaciones empíricas a partir de un conjunto de datos y, por último, en modelos con apoyo de relaciones empíricas.

Entre las diferentes aproximaciones al escalado, algunos autores han planteado sin desarrollar totalmente, una aproximación fundamental, que involucra el modelado del proceso en consideración. El modelo obtenido debe proporcionar un buen entendimiento del proceso para permitir su escalado de forma confiable. Se resalta que en este procedimiento, la validación del modelo a las diferentes escalas y regímenes de operación es la tarea crítica. Johnstone y Thring (1957) plantean que los factores de escala se pueden deducir de un modelo de proceso lo suficientemente preciso. Selman (1983) menciona las relaciones entre grupos adimensionales como constructoras de modelos que deben conducir a establecer un formalismo (un procedimiento formal) para un escalado más preciso. Bisio y Kabel (1985) afirman que procesos que involucran un gran número y complejidad de transformaciones serían mejor escalados si se tiene un modelo apropiado delos mismos. Finalmente, todos los autores concluyen que en escalado, como alternativa para la experimentación y para el análisis dimensional, se encuentra el análisis matemático de un modelo que represente fielmente el proceso. Esto deja abierta la puerta para explorar una metodología para el escalado de procesos desde su modelo matemático. Justamente es eso lo que se desarrolla en este trabajo.

Comúnmente, el escalado de procesos se ha llevado a cabo a través de procedimientos altamente heurísticos. Los números adimensionales y los factores controlantes han marcado tendencias repetidas, generalmente sin éxito completo en el escalado final (Bisio y Kabel, 1985; Rosabal, 1988; Lemus y otros, 1998, Wal et al, 2001). Sin embargo, ya desde la década del 50 del siglo XX se ha escrito sobre la opción de escalar los procesos contando con un modelo lo suficientemente preciso para tal fin. Tal aproximación presenta una ventaja principal: mantener el Punto de Operación $(\mathrm{PdeO})$ y el Régimen de Operación $(\mathrm{RdeO})$ del proceso en la nueva escala, lo que los otros métodos no consideran de manera simultánea. En la literatura (Bisio y Kabel, 1895) se define el escalado como la "operación y puesta en marcha de manera exitosa de una unidad de tamaño comercial cuyo diseño y procedimientos de operación se basan, en parte, en experimentación y demostración a una escala más pequeña de operación". Lo "exitoso" en esta definición indica la producción del producto a las velocidades planeadas, con los costos de manufactura proyectados y cumpliendo con los estándares de calidad deseados. Por su parte, en la definición que da Kossen (1996) al escalado, se cambia la concepción errada de asociar el escalado con el simple aumento de las dimensiones de un equipo de proceso: "El escalado es el diseño y construcción de una unidad o conjunto de unidades de procesamiento a gran escala, tomando como base los cálculos y experimentos a pequeña escala". Esto tampoco debe llevar a que el problema de escalado, dada una capacidad de producción requerida, se soluciona utilizando un gran número de dispositivos idénticos de pequeña escala. Tal solución puede no ser la de mínimo costo.

El escalado por métodos tradicionales presenta múltiples problemas: cambios en los tiempos de respuesta del proceso, aparición de fenómenos superficiales no considerados en la baja escala, aparición de comportamientos cinéticos no contemplados, poca especificación del flujo de fluidos y de los efectos de los cambios en las materias primas sobre el producto (Kossen, 1996). Estos problemas solamente se detectan cuando la interacción de los fenómenos de transferencia de masa, energía y cantidad de movimiento se altera con el cambio de escala. Tal interacción es la que permite distinguir un $\mathrm{PdeO}$, y usarse para caracterizar un $\mathrm{RdeO}$ del proceso. Los métodos reportados para el escalado de procesos, hasta ahora no presentan una metodología que permita 
aumentar la escala de producción desde el laboratorio a planta piloto o a nivel comercial directamente, de manera eficiente y eficaz. Esta dificultad puede residir en el cambio de los RdeO en la nueva escala. En dichos métodos, a tales cambios no se les presta atención por intentar mantener el $\mathrm{PdeO}$, incluso a costa de sobrediseños y sobrecostos en la operación del proceso. Todo esto invita a una consideración más profunda de tal sacrificio en los RdeO originales del proceso cuando se va a la nueva escala. El método presentado en este trabajo incorpora una herramienta de la teoría de control para determinar el RdeO, de modo que conociendo el PdeO deseado, el proceso en la nueva escala pueda ser ubicado en un entorno pequeño de tal PdeO pero manteniendo el RdeO.

Este trabajo está organizado como sigue: en la sección 2 se presenta el problema de escalado de procesos, en la sección 3 se definen el Punto de Operación (PdeO) y el Régimen de Operación (RdeO) como conceptos fundamentales para discutir el escalado. En la sección 4 se expone la forma de determinar la jerarquía de dinámicas en un proceso, con el fin de proponer en la sección 5 un método de escalado basado en dicha jerarquía y mostrar un ejemplo de aplicación. Finalmente, la sección 6 presenta las conclusiones.

\section{PUNTO DE OPERACIÓN Y RÉGIMEN DE OPERACIÓN DE UN PROCESO}

Como ya se dijo, el Punto de Operación (PdeO) y el Régimen de Operación (RdeO) son conceptos fundamentales para discutir el escalado de procesos. Por eso, y debido a la ausencia de definiciones claras en la literatura para estos dos conceptos, en lo que sigue se proponen dos definiciones útiles.

\section{Punto de Operación (PdeO) de un Proceso}

El PdeO hace referencia a las características físicas y químicas que definen el estado del sistema. Es decir, el PdeO debería definirse a través del vector de estado del proceso. La definición de vector de estado, o simplemente estado, se toma de Kalman (1960), debido a su uso exitoso en teoría de control, pero con una restricción adicional de interpretabilidad, como se ve en la siguiente definición.

\section{Definición 1}

El Punto de Operación (PdeO) está dado por las variables del vector de estado del proceso, siempre y cuando todas las variables de estado tengan interpretación de acuerdo con la definición y operación del proceso. Las variables de estado son el conjunto más pequeño de variables $\mathbf{x}$, de modo que conocido su valor en el instante inicial $\mathbf{x}\left(\mathrm{t}_{0}\right)$ y las entradas al proceso $u(t)$ desde $t_{0}$ hasta $t$, es posible predecir el valor del estado en el instante t. El PdeO es un valor dado de las variables de estado que además constituye un punto de equilibrio del sistema matemático que describe al proceso: la derivada de $\mathrm{x}$ respecto a $\mathrm{t}$ es igual a cero con $\mathrm{u}=\mathrm{u}_{\text {equilibrio }}=$ constante. $\mathrm{El} \mathrm{PdeO}$ es un punto en $\mathrm{R}^{\mathrm{n}}$, con $\mathrm{n}$ la dimensión del espacio de estados del proceso.

Puesto que los estados deben cumplir con la restricción mencionada, es necesario que su deducción provenga de un Modelo Semifísico de Base Fenomenológica (MSBF) del proceso (Alvarez et al., 2009). La base de los MSBF es la aplicación del principio de conservación (materia, energía y cantidad de movimiento) al proceso. En consecuencia, las variables que aparecen en los diferenciales de las ecuaciones del modelo pueden tomarse directamente como los estados $(\mathbf{x})$ del proceso. De este modo, su interpretación está garantizada desde la deducción misma del modelo, al aplicar balances de masa, energía y cantidad de movimiento en estado transitorio. Es así como los estados logran caracterizar los efectos de interés del proceso.

Debe recalcase que en el método de escalado aquí propuesto no se busca mantener el valor de todas las variables en la escala inicial. Por el contrario, se permite que las variables de estado (x) puedan moverse en un intervalo pequeño alrededor de los valores originales de cada variable en la escala inicial. Además, en el caso del escalado, se toma por analogía u como el vector de parámetros de diseño. 


\section{Régimen de Operación (RdeO)}

Respecto a Régimen de Operación (RdeO), no se ha encontrado en la literatura una definición general y común a diferentes autores. A partir de la revisión bibliográfica de este concepto se propone aquí una definición con el objeto de su aplicación al escalado de procesos a partir del MSBF. La definición de RdeO que aquí se presenta está asociada con todos los efectos dinámicos involucrados (de interés) en el proceso. Estos efectos son consecuencia del movimiento del fluido, el intercambio de energía en el sistema como un todo, la transferencia de masa y las reacciones químicas y bioquímicas presentes. Por tanto, los efectos se asocian y se describen a partir de los estados del proceso, que como ya se dijo aparecen naturalmente de las ecuaciones de balance que dan estructura al modelo.

\section{Definición 2}

El Régimen de Operación (RdeO) es una relación de jerarquía, que se establece de acuerdo con un criterio o característica dada, entre los efectos dinámicos que coexisten en el sistema en el $\mathrm{PdeO}$ y que caracterizan y definen el comportamiento del proceso en un punto específico y en un entorno que lo contenga. La jerarquía se establece entre cada uno de los efectos individuales del proceso, que no son más que el conjunto de estados $(\mathbf{x})$.

\section{JERARQUÍA DE DINÁMICAS EN UN PROCESO}

La clave del método de escalado que aquí se propone es la definición de RdeO, que se estableció previamente, de acuerdo con un criterio o característica dada. En el método propuesto, se utiliza como criterio de jerarquización en la definición del RdeO el Índice de Estado Impactable (IEI), definido tras operar con la matriz de Hankel $(\mathbf{H})$ del modelo del proceso. Por eso son necesarias las siguientes definiciones adicionales.

\section{Definición 3}

La matriz Hankel de un sistema dinámico es la respuesta impulso del sistema, y se calcula a partir de los parámetros de Markov, con las matrices A, B y C de la linealización del sistema como: $h_{i}=$ CA $^{i-1} \mathbf{B}$ con $i=1,2, \ldots n$, siendo $n$ el orden del sistema o dimensión del vector de estados (x). En consecuencia, $\mathrm{H}$ está dada por:

$$
\mathbf{H}=\left[\begin{array}{ccc}
h_{i} & h_{i+1} & h_{i+j} \\
h_{i+1} & h_{i+2} & \\
h_{i+j} & & h_{i+2 j}
\end{array}\right]
$$

Fácilmente se demuestra una forma alternativa, bastante útil: $\mathrm{H}=\mathrm{O}_{b} \mathrm{C}_{0}$, siendo $\mathrm{O}_{b}$ la matriz de observabilidad y $\mathrm{C}_{\mathrm{o}}$ la matriz de controlabilidad del sistema, que se formulan como:

$$
\begin{aligned}
& \mathbf{O}_{\mathrm{b}}=\left[\begin{array}{llll}
\mathbf{C} & \mathbf{C A} & \ldots & \mathrm{CA}^{\mathrm{n}-1}
\end{array}\right]^{\top} \\
& \mathrm{C}_{\mathrm{o}}=\left[\begin{array}{llll}
\mathbf{B} & \mathbf{A B} & \ldots & \mathbf{A}^{\mathrm{n}-1} \mathrm{~B}
\end{array}\right]
\end{aligned}
$$

Las matrices de observabilidad y de controlabilidad se obtienen a partir del sistema representado por las ecuaciones (4) y (5). 
$\mathbf{x}(\mathrm{k}+1)=\mathbf{A}_{\mathrm{d}} \mathbf{x}(\mathrm{k})+\mathbf{B}_{\mathrm{d}} \mathbf{u}(\mathrm{k})$

$\mathbf{y}(\mathrm{k})=\mathbf{C x}(\mathrm{k})$

Donde $\mathrm{x}(\mathrm{k}) \in \mathrm{R}^{\mathrm{n}}$. $\mathrm{A}_{d} \mathrm{y} \mathrm{B}_{d}$ son las matrices del modelo discreto de sistema, nótese que la matriz $\mathrm{C}$ permanece igual al caso del modelo continuo. Además, el sistema será observable si hay un $\mathrm{k}$ finito tal que el conocimiento de las entradas $u(0), u(1), \ldots u(k-1)$ y de las salidas $y(0), y(1), \ldots y(k-1)$ es suficiente para determinar el estado inicial del sistema. Para simplificar se asume que $u(k)=0$ y que $\mathrm{y}(0), \mathrm{y}(1), \ldots \mathrm{y}(\mathrm{n}-1)$ son conocidos. De ahí puede escribirse el siguiente conjunto de ecuaciones:

$\mathbf{y}(0)=\mathbf{C x}(0)$

$\mathbf{y}(1)=\mathbf{C x}(1)=\mathbf{C A}_{\mathrm{d}} \mathbf{x}(0)$

$y(2)=\mathbf{C x}(2)=\mathrm{CA}_{\mathrm{d}}{ }^{2} \mathbf{x}(0)$

$y(n-1)=C x(n-1)=C A_{d}{ }^{n-1} x(0)$

Usando notación vectorial, se obtiene:

$\left[\begin{array}{c}\mathbf{y}(0) \\ \mathbf{y}(1) \\ \vdots \\ \mathbf{y}(n-1)\end{array}\right]=\left[\begin{array}{c}\mathbf{C} \\ \mathbf{C A} \\ \vdots \\ \mathbf{C A}_{d}{ }^{n-1}\end{array}\right] \mathbf{x}(0)$

La matriz obtenida es la matriz de observabilidad, y representa la relación entre el estado inicial $x(0)$ y la secuencia de mediciones de la salida $y(0), y(1), \ldots y(n-1)$ (Botero y Álvarez, 2009).

Para la obtención de la matriz de controlabilidad, suponga que el estado inicial $\mathbf{x}(0)$ está dado:

$\mathbf{x}(1)=\mathbf{A}_{\mathrm{d}} \mathbf{x}(0)+\mathbf{B}_{\mathrm{d}} \mathbf{u}(0)$

$\mathbf{x}(2)=\mathbf{A}_{\mathrm{d}} \mathbf{x}(1)+\mathbf{B}_{\mathrm{d}} \mathbf{u}(1)=\mathbf{A}_{\mathrm{d}}{ }^{2} \mathbf{x}(0)+\mathbf{A}_{\mathrm{d}} \mathbf{B}_{\mathrm{d}} \mathbf{u}(0)+\mathbf{B}_{\mathrm{d}} \mathbf{u}(1)$

$\mathbf{x}(3)=\mathbf{A}_{\mathrm{d}} \mathbf{x}(2)+\mathbf{B}_{\mathrm{d}} \mathbf{u}(2)=\mathbf{A}_{\mathrm{d}}{ }^{3} \mathbf{x}(0)+\mathbf{A}_{\mathrm{d}}{ }^{2} \mathbf{B}_{\mathrm{d}} \mathbf{u}(0)+\mathbf{A}_{\mathrm{d}} \mathbf{B}_{\mathrm{d}} \mathbf{u}(1)+\mathbf{B}_{\mathrm{d}} \mathbf{u}(2)$

$\mathrm{Y}$ siguiendo sucesivamente el desarrollo indicado, el estado en el instante $\mathrm{n}$, donde $\mathrm{n}$ es el orden del sistema, está dado por:

$\mathbf{x}(n)=A_{d}^{n} \mathbf{x}(0)+A_{d}^{n-1} B_{d} \mathbf{u}(0)+A_{d}^{n-2} B_{d} \mathbf{u}(1)+\ldots+B_{d} \mathbf{u}(n-1)$

En notación vectorial, puede expresarse como: 
$\mathbf{x}(n)=\mathbf{A}_{d}{ }^{n} \mathbf{x}(0)+\left[\begin{array}{llll}\mathbf{B}_{d} & \cdots & \mathbf{A}_{d}{ }^{n-2} \mathbf{B}_{d} & \mathbf{A}_{d}{ }^{n-1} \mathbf{B}_{d}\end{array}\right]\left[\begin{array}{c}\mathbf{u}(\mathrm{n}-1) \\ \vdots \\ \mathbf{u}(1) \\ \mathbf{u}(0)\end{array}\right]$

La matriz que relaciona la secuencia de entradas pasadas $u(n-1), \ldots, u(1), u(0)$ con el estado actual $x(n)$, representa la matriz de controlabilidad. Nótese que el primer término de esta expresión es constante.

Para la obtención de la matriz de Hankel, por simplicidad, de aquí en adelante se suprimirá el subíndice $d$ en las matrices $A$ y $B$, que indica que la matriz corresponde al sistema discreto. En la expresión de observabilidad en lugar de evaluar el estado inicial en 0 , se evaluará en el instante $n$ :

$\left[\begin{array}{c}y(n) \\ y(n+1) \\ \vdots \\ y(2 n-1)\end{array}\right]=\left[\begin{array}{c}C \\ C A \\ \vdots \\ C^{n-1}\end{array}\right] x(n)$

Ahora, en esta expresión se reemplaza x(n) de la expresión de la controlabilidad.

$\left[\begin{array}{c}\mathbf{y}(n) \\ \mathbf{y}(n+1) \\ \vdots \\ \mathbf{y}(2 n-1)\end{array}\right]=\left[\begin{array}{c}\mathbf{C} \\ \mathbf{C A} \\ \vdots \\ \mathbf{C A}^{\mathrm{n}-1}\end{array}\right] \mathbf{A}^{\mathrm{n}} \mathbf{x}(0)+\left[\begin{array}{c}\mathbf{C} \\ \mathbf{C A} \\ \vdots \\ \mathbf{C A}^{\mathrm{n}-1}\end{array}\right]\left[\begin{array}{llll}\mathbf{B} & \ldots & \mathbf{A}^{\mathrm{n}-2} \mathbf{B} & \mathbf{A}^{\mathrm{n}-1} \mathbf{B}\end{array}\right]\left[\begin{array}{c}\mathbf{u}(\mathrm{n}-1) \\ \vdots \\ \mathbf{u}(1) \\ \mathbf{u}(0)\end{array}\right]$

Nótese que el primer término de la expresión anterior es constante, y el segundo término representa la relación dinámica entre las entradas anteriores al instante $\mathrm{n}$ y las salidas posteriores al instante $\mathrm{n}$. Dicha relación está dada por el producto de las matrices de observabilidad y controlabilidad, más conocida como la matriz de Hankel.

$H=\left[\begin{array}{c}C \\ C A \\ \vdots \\ C^{n-1}\end{array}\right]\left[\begin{array}{llll}B & \cdots & A^{n-2} B & A^{n-1} B\end{array}\right]$

$H=\left[\begin{array}{cccc}C B & C A B & \cdots & C A^{n-1} B \\ C A B & C A^{2} B & \cdots & C A^{n} B \\ \vdots & \vdots & & \vdots \\ C A^{n-1} B & C A^{n} B & \cdots & C A^{2 n-2} B\end{array}\right]$

Definiendo K como:

$K=\left[\begin{array}{c}C \\ C A \\ \vdots \\ C A^{n-1}\end{array}\right] A^{n} \times(0)$ 


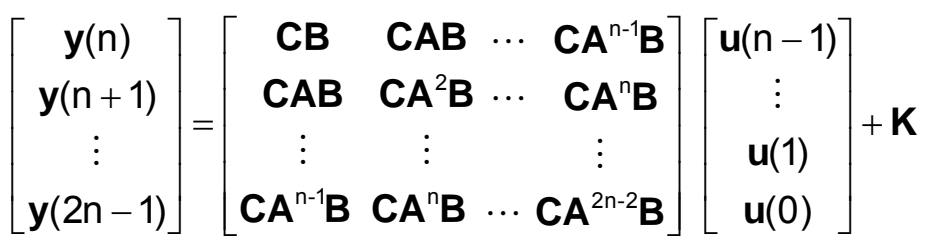

$\left[\begin{array}{c}\mathbf{y}(n) \\ \mathbf{y}(n+1) \\ \vdots \\ \mathbf{y}(2 \mathrm{n}-1)\end{array}\right]=\mathbf{H}\left[\begin{array}{c}\mathbf{u}(\mathrm{n}-1) \\ \vdots \\ \mathbf{u}(1) \\ \mathbf{u}(0)\end{array}\right]+\mathbf{K}$

La ecuación (15) muestra claramente que la matriz de Hankel $(H)$ es una representación dinámica del sistema, a través de la cual se obtiene la secuencia de respuestas a partir del instante n: $(n)$, $y(n+1), \ldots y(2 n-1)$, ante una secuencia de entradas previas al instante $n: u(0), u(1), \ldots, u(n-1)$. Debe comentarse que aunque esta es la respuesta del sistema linealizado, para el uso de $\mathrm{H}$ en el escalado de procesos, se considera que el proceso operará en un punto de operación definido. Como se sabe, siempre la linealización y el sistema no lineal original coinciden justo en el punto en el que se linealiza. Por lo tanto, la linealización se debe realizar alrededor del punto de operación esperado para el proceso. Esto no representa ninguna limitación en el método puesto que las variables de escala se consideran aplicables en el punto de operación seleccionado. Nótese además, que la matriz de Hankel tiene orden n.lx n.m, donde $n$ es el orden del sistema, $m$ es el número de entradas y I es el número de salidas. Si el número de entradas es igual al de salidas, I=m, la matriz de Hankel será una matriz cuadrada de orden n.m.

$\mathrm{H}$ representa las propiedades entrada-salida del sistema en términos dinámicos y ha sido ampliamente estudiada en el análisis de sistemas lineales (Moore, 1981; Wal y Jager, 2001). En particular, los valores singulares de $\mathrm{H}$ tienen un significado importante en la teoría de sistemas lineales (Kailath, 1980). Estos se calculan como la raíz cuadrada de los valores propios de $\mathrm{H}$. Dentro de las principales aplicaciones de la descomposición en valores singulares (SVD por su sigla en inglés) de $\mathrm{H}$ está la reducción de modelos, la identificación de sistemas y el diseño de filtros digitales (Wal y Jager, 2001). Por su parte, Van de Wal y De Jager (2001) consideran que los valores singulares de $\mathrm{H}$ están asociados a la controlabilidad de estado en un sentido cuantitativo, aspecto que ya ha sido explotado para diseñar estructuras de control (Álvarez, 2008), y que en el presente trabajo se usa para obtener la jerarquización de los estados del proceso que se escala.

El procedimiento es como sigue: se aplica SVD a la matriz de Hankel para llegar a $H=U \Sigma V^{\top}$, con los vectores columna de $\mathrm{U}$ y de $\mathrm{V}$ ortogonales y ortonormales y $\Sigma$ una matriz diagonal que contiene los valores singulares de $\mathrm{H}$ y por lo tanto del proceso. El número de vectores singulares que se tomen es igual al número de valores singulares considerados para aproximar la matriz original. Por simplicidad, lo más frecuente es tomar únicamente el primer valor singular para la aproximación. Existen casos en los que ese grado de aproximación resulta bajo, por lo que se deben incluir más vectores para la aproximación.

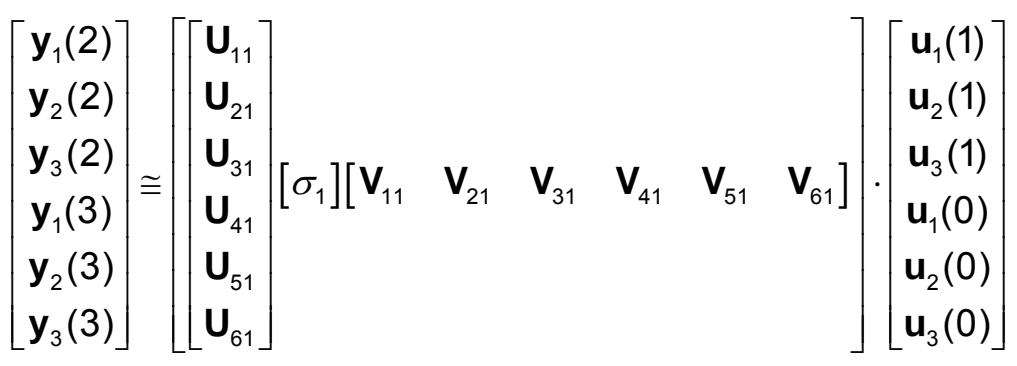


Al desarrollar completamente la ecuación, se obtiene un vector en el cual las salidas están expresadas en función de las entradas y de los valores obtenidos en la descomposición SVD. En esta expresión es posible visualizar el papel de cada elemento de los vectores singulares y del valor singular tomado:

$$
\left[\begin{array}{l}
\mathbf{y}_{1}(2) \\
\mathbf{y}_{2}(2) \\
\mathbf{y}_{3}(2) \\
\mathbf{y}_{1}(3) \\
\mathbf{y}_{2}(3) \\
\mathbf{y}_{3}(3)
\end{array}\right] \cong\left[\begin{array}{l}
\mathbf{U}_{11} \sigma_{1}\left(\mathbf{V}_{11} \mathbf{u}_{1}(1)+\mathbf{V}_{21} \mathbf{u}_{2}(1)+\mathbf{V}_{31} \mathbf{u}_{3}(1)+\mathbf{V}_{41} \mathbf{u}_{1}(0)+\mathbf{V}_{51} \mathbf{u}_{2}(0)+\mathbf{V}_{61} \mathbf{u}_{3}(0)\right) \\
\mathbf{U}_{21} \sigma_{1}\left(\mathbf{V}_{11} \mathbf{u}_{1}(1)+\mathbf{V}_{21} \mathbf{u}_{2}(1)+\mathbf{V}_{31} \mathbf{u}_{3}(1)+\mathbf{V}_{41} \mathbf{u}_{1}(0)+\mathbf{V}_{51} \mathbf{u}_{2}(0)+\mathbf{V}_{61} \mathbf{u}_{3}(0)\right) \\
\mathbf{U}_{31} \sigma_{1}\left(\mathbf{V}_{11} \mathbf{u}_{1}(1)+\mathbf{V}_{21} \mathbf{u}_{2}(1)+\mathbf{V}_{31} \mathbf{u}_{3}(1)+\mathbf{V}_{44} \mathbf{u}_{1}(0)+\mathbf{V}_{51} \mathbf{u}_{2}(0)+\mathbf{V}_{61} \mathbf{u}_{3}(0)\right) \\
\mathbf{U}_{41} \sigma_{1}\left(\mathbf{V}_{11} \mathbf{u}_{1}(1)+\mathbf{V}_{21} \mathbf{u}_{2}(1)+\mathbf{V}_{31} \mathbf{u}_{3}(1)+\mathbf{V}_{41} \mathbf{u}_{1}(0)+\mathbf{V}_{51} \mathbf{u}_{2}(0)+\mathbf{V}_{61} \mathbf{u}_{3}(0)\right) \\
\mathbf{U}_{51} \sigma_{1}\left(\mathbf{V}_{11} \mathbf{u}_{1}(1)+\mathbf{V}_{21} \mathbf{u}_{2}(1)+\mathbf{V}_{31} \mathbf{u}_{3}(1)+\mathbf{v}_{41} \mathbf{u}_{1}(0)+\mathbf{V}_{51} \mathbf{u}_{2}(0)+\mathbf{V}_{61} \mathbf{u}_{3}(0)\right) \\
\mathbf{U}_{61} \sigma_{1}\left(\mathbf{V}_{11} \mathbf{u}_{1}(1)+\mathbf{V}_{21} \mathbf{u}_{2}(1)+\mathbf{V}_{31} \mathbf{u}_{3}(1)+\mathbf{V}_{41} \mathbf{u}_{1}(0)+\mathbf{V}_{51} \mathbf{u}_{2}(0)+\mathbf{V}_{61} \mathbf{u}_{3}(0)\right)
\end{array}\right]
$$

Nótese que según la ecuación (17), en cada salida no es posible discriminar una entrada que tenga impacto diferente, en relación a las demás salidas. Esto se debe a que la expresión que contiene las entradas es la misma para todas las salidas:

$\left(\mathbf{V}_{11} u_{1}(1)+\mathbf{V}_{21} u_{2}(1)+\mathbf{V}_{31} u_{3}(1)+\mathbf{V}_{41} u_{1}(0)+\mathbf{V}_{51} u_{2}(0)+\mathbf{V}_{61} u_{3}(0)\right)$

Lo único que diferencia a las salidas entre sí son los valores del vector de la descomposición SVD asociado a las salidas: $U_{i 1}$. Esto significa que el análisis permite determinar el impacto de cada entrada sobre todo el proceso, sin discriminar entre las salidas, y este impacto está dado solamente por el valor de $\mathrm{V}_{\mathrm{j} 1}$. De la misma manera, para cada entrada manipulada no es posible determinar la salida a la cual más impacta, en relación a las demás entradas. Puesto que las salidas están ponderadas por un valor $U_{i 1}$ que multiplica al término que contiene las entradas. Por lo tanto, sólo es posible determinar a través de los valores de $U_{i 1}$ el impacto neto de las entradas sobre cada salida.

Operando con las matrices $U, V$ y $\Sigma$, se pueden encontrar cuales estados del proceso son más impactables respecto de las entradas como un todo, a través de un índice cuantitativo que permite establecer un orden o jerarquía de las dinámicas. El efecto de las entradas como un todo sobre la salida i, denotado como $\alpha_{y i}$, se calcula con base en los vectores de salida $U_{i 1}$, y da una medida de qué tan impactable dinámicamente es la salida i en el proceso. Por simplicidad, sin que eso genere ninguna restricción, en este trabajo se considera que los estados son medidos o estimados, por lo que cada salida representa un estado $\mathrm{y}_{\mathrm{i}}=\mathrm{x}_{\mathrm{i}}$ (Botero y Álvarez, 2011).

\section{Definición 4}

El Índice de Estado Impactable (IEI) indica que tan impactable es cada estado desde la entrada como un todo. Si se asume que todos los estados están medidos, se cumple que $\mathrm{y}_{\mathrm{i}}=\mathrm{x}_{\mathrm{i}}$, con $\mathrm{i}=1,2, \ldots \mathrm{n}$, y por lo tanto se puede denominar a $\alpha_{\mathrm{yi}}$ el IEI del estado i. En un sistema con $\mathrm{n}$ estados, ny salidas y nu entradas, para la k-ésima variable su IEI se calcula como:

$\mid \mathrm{IEI}_{\mathrm{k}}=\sqrt{\sum_{\mathrm{i}}\left[\sigma_{\mathrm{i}}^{2} \sum_{\mathrm{j}=0}^{\mathrm{n}-1}\left[\mathbf{U}^{2}{ }_{\left(\mathrm{k}+\mathrm{n} \mathbf{y}^{*} \mathrm{j}\right), \mathrm{i}}\right]\right]}$

Con $\sigma_{i}$ definido como el i-ésimo valor singular. EI IEI es útil para determinar la relevancia de las dinámicas más impactables del sistema con el cambio de escala. Es decir, permite determinar la jerarquía de las dinámicas con lo cual se define el RdeO, que se especifica como el orden de las dinámicas del proceso, empezando con la que mayor IEI tenga y terminando con la dinámica con el menor IEl. 


\section{MÉTODO DE ESCALADO Y EJEMPLO DE APLICACIÓN}

Para establecer el método de escalado que aquí se propone, es necesario definir el valor mínimo de capacidad del proceso a partir del cual se puede escalar.

\section{Definición 5}

La Variable de Capacidad (VdeC) del proceso, en su valor crítico (VdeC*) es el valor de capacidad a partir del cual la dominancia de la dinámica deseada es superior a otras dinámicas relevantes en el proceso. En ese punto interactúan todas las dinámicas del sistema, lo cual garantiza la presencia de todos los efectos fisicoquímicos y bioquímicos que se van a tener en cuenta a otras escalas.

En la aplicación de la analogía de la Jerarquización dinámica basada en SVD de la matriz de Hankel para el escalado de procesos, el VdeC* se da cuando aparece un valor mayor del IEI para la dinámica de interés, respecto a las otras dinámicas importantes del sistema. Es posible que otras dinámicas no relevantes en el resultado esperado del proceso presenten un IEl superior al de la dinámica de interés, pero esta última deberá superar sólo las demás dinámicas relevantes en el sistema. En este caso, la presencia de un IEI superior al de la dinámica de interés, para dinámicas no relevantes en el sistema, indica la importancia de tener en cuenta todas las dinámicas presentes en el sistema e identificar su influencia al cambiar de escala, con lo cual se ganará conocimiento del proceso desde los resultados matemáticos obtenidos con las condiciones de operación en una primera escala de proceso. La relevancia o no de una dinámica se considera en este trabajo directamente asociada con su influencia sobre la calidad del producto del proceso. Nótese que la relevancia no indica de manera directa la jerarquía (el RdeO). Es decir, el deseo del Ingeniero de Proceso no dicta la jerarquía. Tal jerarquía dinámica es propia del proceso y sus interacciones. El método de escalado que aquí se propone consta de nueve pasos principales, que van desde la especificación del proceso hasta la determinación de los valores para las variables de diseño en la nueva escala. El procedimiento, explicado paso a paso y tomando un fermentador como ejemplo, es como sigue:

\section{Paso 1: Especificación del proceso y de la tarea de escalado}

Este primer paso implica especificar el proceso y obtener su Modelo Semifísico de Base Fenomenológica (MSBF): i) definir cuáles serán las variables de estado $\mathbf{x}$ (que especificarán el $\mathrm{PdeO}$ ), cuáles serán variables manipuladas (o de escala) u y cuales variables de diseño $\mathbf{z}$, todas con su valor correspondiente al $\mathrm{PdeO}$ de la Baja Escala $\left(\mathrm{PdeO}_{\mathrm{BE}}\right)$, y ii) declarar el valor de la $V_{d e C}{ }^{*}$ a la que se quiere llegar: $\mathrm{PdeO}$ de la Alta Escala $\left(\mathrm{PdeO}_{\mathrm{AE}}\right)$, y precisar la eficiencia mínima a lograr en ese $\mathrm{PdeO}_{\mathrm{AE}}$.

El proceso tomado como ejemplo en este trabajo es la fermentación continua de un jarabe glucosado, complementado con fosfatos, sales de potasio y fuentes de nitrógeno, cofactores de la ruta metabólica por la cual la levadura Saccharomyce cerevisiae convierte, en fase líquida, la glucosa a etanol y $\mathrm{CO}_{2}$ (Abril y Abril, 2009). Para la fermentación se utiliza un reactor cilíndrico cerrado y enchaquetado, que se agita mediante una turbina tipo Rushton. Esta clase de agitación produce flujos axiales y radiales que garantizan la homogeneidad del sistema. Al comienzo del proceso, la producción de $\mathrm{CO}_{2}$ desplaza el aire presente, lo cual garantiza las condiciones anaerobias propias de la fermentación de etanol con la levadura. El volumen efectivo del medio corresponde al $70 \%$ del volumen total del reactor. En el arranque, el reactor es inoculado con la levadura en su etapa de crecimiento exponencial. Inocular la levadura en esta etapa de crecimiento garantiza las mejores condiciones para la producción de etanol.

El reactor opera en continuo con un flujo de alimentación del jarabe y renovación de células cuando se presente pérdida de viabilidad de éstas. El reactor tiene dos corrientes de salida: una en fase líquida, constituida por el medio de fermentación residual y etanol, y la otra en fase gaseosa, principalmente formada por $\mathrm{CO}_{2}$ y vapor de agua. El modelo se obtiene aplicando el principio de conservación en sólo un sistema de proceso: fase líquida del fermentador. Por esa 
razón, sólo se efectúan balances de masa y energía para el medio sumergido. La nomenclatura de variables es la usual en modelos de bioprocesos. Se remite al lector a Bastin y Docheim (1990), para observar dicha nomenclatura. Las condiciones de operación para la temperatura, el $\mathrm{pH}$ y el nivel de agitación en el proceso se establecen de acuerdo con el metabolismo de la levadura cuando produce etanol a partir de glucosa (Ferreyra y otros, 2009, Araya y otros, 2007, Calderón-Santoyo y otros, 2010, Roeva y otros, 2007).

El vector de estados está formado por: $\mathbf{x}=[\mathrm{X}, \mathrm{P}, \mathrm{S}, \mathrm{T}]$, con $\mathrm{X}, \mathrm{P}, \mathrm{S}$ concentraciones de biomasa, producto y sustrato respectivamente, y $\mathrm{T}$ la temperatura de proceso. Los valores nominales esperados para el PdeO son: $X=20 \mathrm{~kg} / \mathrm{m}^{3}, S=150 \mathrm{~kg} / \mathrm{m}^{3}, P=30 \mathrm{~kg} / \mathrm{m}^{3}$ y $\mathrm{T}=30^{\circ} \mathrm{C}$. Las variables manipuladas u son: $u=\left[F_{i n} C_{v} S_{i n} U_{g}\right]$, con $F_{i n}, C v, S_{\text {in }} y U_{g}$ el flujo de alimentación, el coeficiente de apertura de la válvula de control de nivel, la concentración de sustrato de alimentación y el coeficiente global de transferencia de calor, respectivamente. Finalmente, las variables de diseño $z$ son: $z=\left[F_{\text {in }} X_{\text {in }} S_{\text {in }} P_{\text {in }}\right]$, con $X_{\text {in }} y P_{\text {in }}$ las concentraciones de biomasa y producto en la entrada respectivamente. Nótese que en el vector $z$ existen variables que también figuran en el vector $u$, lo que sugiere que los valores de $F_{\text {in }}$ y $S_{\text {in }}$ que se hallen para las variables de diseño serán los valores nominales (iniciales o bias value) que tomen los controladores de esas variables cuando empiezan a operar en lazo cerrado.

En el caso tratado aquí, se desea escalar hasta un volumen de 1000 litros, por lo que VdeC=1000 I. La eficiencia del proceso esperada es aquella que brinde al menos $30 \mathrm{~kg} / \mathrm{m}^{3}$ de Etanol en estado estacionario (Pee). Para el RdeO se espera que la dinámica dominante del proceso corresponda a la dinámica de la concentración de etanol $(P)$. Finalmente, el MSBF se realiza mediante los balances de masa en el biorreactor, considerando la cantidad de biomasa que entra con el flujo, ante el caso eventual de una renovación celular. También se considera la biomasa generada y la biomasa que pierde viabilidad. Los siguientes son los balances de sustrato biomasa, producto y energía del proceso explícitos, con las ecuaciones constitutivas que explican los términos involucrados en los balances. Todas estas ecuaciones constituyen el Modelo Semifísico de Base Fenomenológica (MSBF) del proceso.

\section{Balance de sustrato}

Considera, además del sustrato que entra y sale, el sustrato consumido para crecimiento y mantenimiento celular. También considera el sustrato convertido en etanol, así como el sustrato que dejan de consumir las células por pérdida de su viabilidad. Es decir, la cinética de producción de etanol considera la viabilidad de todas las células por lo tanto se corrige este término teniendo en cuenta el sustrato que no es convertido a etanol.

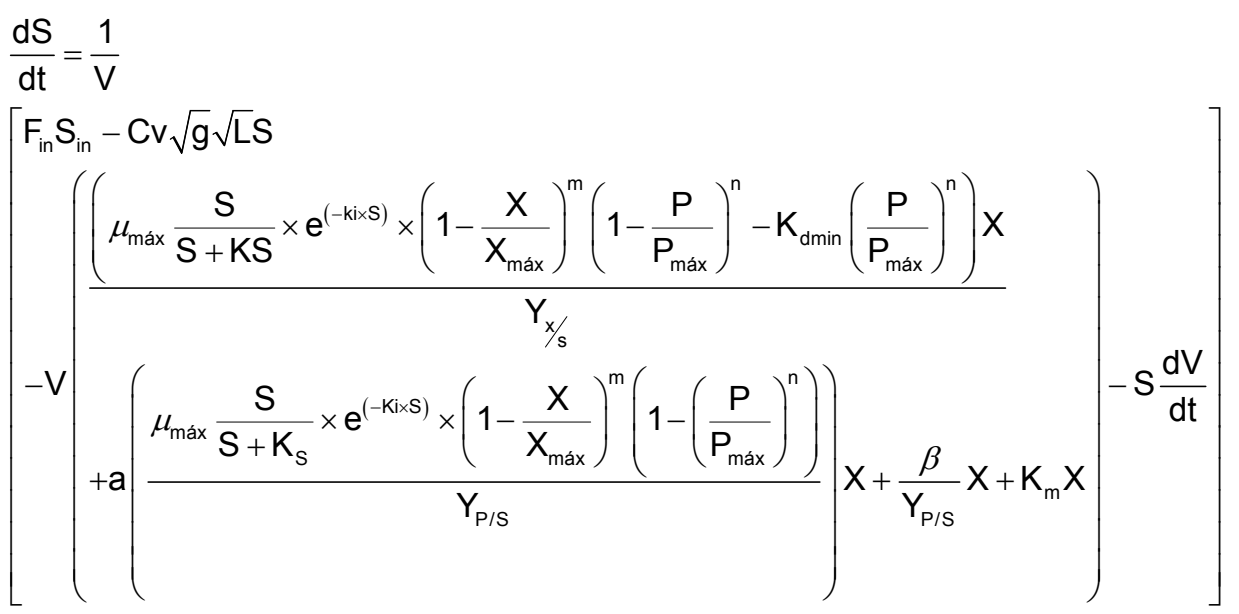

Con $S$ concentración de sustrato en $\left[\mathrm{kg} / \mathrm{m}^{3}\right]$, $\mathrm{P}$ concentración de producto en $\left[\mathrm{kg} / \mathrm{m}^{3}\right]$, $\mathrm{F}$ flujo volumétrico en $\left[\mathrm{m}^{3} / \mathrm{s}\right]$, y $X$ concentración de biomasa en $\left[\mathrm{kg} / \mathrm{m}^{3}\right]$. $X_{\text {máx }}$ y $P_{\text {máx }}$ corresponden a la máxima concentración de biomasa y producto, respectivamente. $Y_{P / S}$ es el rendimiento de $P$ respecto a $\mathrm{S}$. 


\section{Balance de biomasa}

En este balance, se consideró como ecuación constitutiva la velocidad de crecimiento de biomasa propuesta por Monod, pero corregida por efectos de presión y concentración de biomasa. La velocidad máxima de crecimiento celular es función de la velocidad de crecimiento óptimo, $\mathrm{pH}$ y temperatura del medio de fermentación. Esa velocidad máxima de crecimiento es un parámetro empírico, cuyo valor se tomó de Casablancas y otros (1998).

$$
\begin{aligned}
& \frac{d X}{d t}=\frac{1}{V} F_{\text {in }} X_{\text {in }}-\frac{1}{V} C V \sqrt{g} \sqrt{L} X+\frac{V}{V} X \mu_{\text {máx }} \frac{S}{S+K s} e^{\left(-k_{i} S\right)}\left(1-\frac{X}{X_{\text {máx }}}\right)^{m}\left(1-\frac{P}{P_{\text {máx }}}\right)^{n} \\
& -\frac{V}{V} X K_{\text {dmin }}\left(\frac{P}{P_{\text {máx }}}\right)^{n}-\frac{X}{V} \frac{d V}{d t}
\end{aligned}
$$

\section{Balance de producto}

Considera el producto que entra, porque la velocidad de generación de producto está directamente asociada al crecimiento de la célula. También incluye la velocidad de generación de producto por la reacción metabólica y la cantidad de producto que no se genera por pérdida de viabilidad celular, de forma análoga al balance de sustrato.

$$
\frac{\mathrm{dP}}{\mathrm{dt}}=\frac{1}{\mathrm{~V}}\left[\begin{array}{l}
\mathrm{F}_{\text {in }} \mathrm{P}_{\text {in }}-\mathrm{C} \mathrm{V} \sqrt{\mathrm{g}} \sqrt{\mathrm{LP}}+ \\
\mathrm{V}\left(\alpha\left(\mu_{\text {max }} \frac{\mathrm{S}}{\mathrm{S}+\mathrm{K}_{\mathrm{S}}} \mathrm{e}^{(-\mathrm{ki} \times \mathrm{S})}\left(1-\frac{\mathrm{X}}{\mathrm{X}_{\text {máx }}}\right)^{\mathrm{m}}\left(1-\frac{\mathrm{P}}{\mathrm{P}_{\text {máx }}}\right)^{\mathrm{n}}\right)+\beta\right) \mathrm{X} \\
-\mathrm{VK} \mathrm{K}_{\mathrm{d}} \mathrm{P}-\mathrm{P} \frac{\mathrm{dV}}{\mathrm{dt}}
\end{array}\right]
$$

\section{Balance de energía}

Considera la energía que entra y sale con los flujos y la generación e intercambio de energía con el medio.

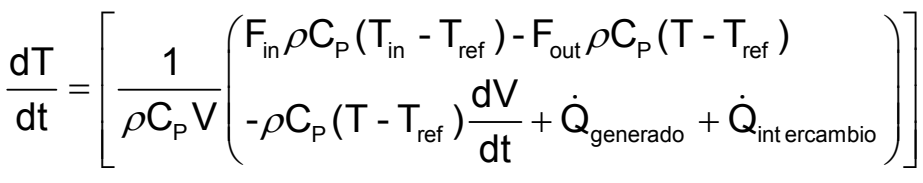

$$
\begin{aligned}
& \dot{\mathrm{Q}}_{\text {generado }}=\frac{\mathrm{V} \mu \mathrm{X}}{\mathrm{Y}_{\Delta}}
\end{aligned}
$$

$\dot{\mathrm{Q}}=\mathrm{F}_{\mathrm{J}} \rho_{\mathrm{J}} \mathrm{C}_{\mathrm{PJ}}\left(\mathrm{T}_{0 J}-\mathrm{T}\right)\left[1-\exp \left(-\frac{\mathrm{U}_{\mathrm{g}} \mathrm{A}_{\mathrm{J}}}{\mathrm{F}_{\mathrm{J}} \rho_{\mathrm{J}} \mathrm{C}_{\mathrm{PJ}}}\right)\right]$

$\mathrm{U}_{g}=\frac{1}{\frac{1}{\mathrm{~h}_{\mathrm{i}}}+\frac{\mathrm{e}}{\kappa}+\frac{1}{\mathrm{~h}_{\mathrm{e}}}}$

donde: $h_{i}$ Coeficiente convectivo en el lado del caldo $\left(\mathrm{W} / \mathrm{m}^{2} \mathrm{~K}\right)$, he Coeficiente convectivo en el lado del fluido $\left(\mathrm{W} / \mathrm{m}^{2} \mathrm{~K}\right)$ y e Espesor de la pared de intercambio $(\mathrm{m})$. 
Paso 2: Calcular la matriz de Hankel para el proceso

Para esto se debe primero linealizar el MSBF del Proceso y llevarlo a la forma canónica:

$\dot{x}=A x+B u ; \quad y=C x$

y con ese modelo formular la Matriz de Hankel $\mathrm{H}$. Para linealizar, se deriva respecto a cada uno de los estados y variables reales manipuladas, considerando $f$ como el lado derecho de cada Ecuación Diferencial del MSBF. De este modo se llega a las matrices $A$ y $B$, mientras que $C$ se deduce desde la configuración de sensores instalada en el proceso. Para la consideración hecha en todo este trabajo, $\mathrm{y}_{\mathrm{i}}=\mathrm{x}_{\mathrm{i}}, \mathrm{C}$ es la matriz identidad.

La matriz $\mathrm{H}$ que se obtiene para el fermentador no se muestra aquí por cuestiones de espacio. Basta con decir que es una matriz de funciones. Para ilustrar esto, se presenta a continuación de manera expandida sólo una parte de la primera columna de $\mathrm{H}$ :

$\mathbf{H}=\left[\begin{array}{ccccccc}\frac{1}{\mathrm{~V}}{ }^{*} \mathrm{X}_{\text {in }} & \mathrm{h}_{1,2} & 0 & 0 & \mathbf{C A B} & \mathbf{C A}^{2} \mathbf{B} & \mathbf{C A}^{3} \mathbf{B} \\ \frac{1}{\mathrm{~V}}{ }^{*} \mathrm{P}_{\text {in }} & \mathrm{h}_{2,2} & 0 & 0 & \mathbf{C A}^{2} \mathbf{B} & \mathbf{C A}^{3} \mathbf{B} & \mathbf{C A}^{4} \mathbf{B} \\ \frac{1}{\mathrm{~V}}{ }^{*} \mathrm{~S}_{\text {in }} & \mathrm{h}_{3,2} & \mathrm{~h}_{3,3} & 0 & \mathbf{C A}^{3} \mathbf{B} & \mathbf{C A}^{4} \mathbf{B} & \mathbf{C A}^{5} \mathbf{B} \\ \mathrm{h}_{4,1} & \mathrm{~h}_{4,2} & 0 & \mathrm{~h}_{4,4} & & & \mathbf{C A}^{2 n-2} \mathbf{B} \\ \mathbf{C A B} & & & & & & \\ \mathbf{C A} A^{2} \mathbf{B} & & & & & & \\ \mathbf{C A} A^{3} \mathbf{B} & & & & & & \end{array}\right]$

Paso 3: Construir la curva de jerarquía de dinámicas con datos de proceso en la baja escala

Se incrementa la VdeC del proceso desde su valor de Baja Escala (VdeC $\mathrm{BE}_{\mathrm{BE}}$ ) hasta un valor deseado de Alta Escala $\left(V_{d e} C_{A E}\right)$, calculando para cada nuevo valor de la VdeC el IEI de cada estado. Se utilizan para eso los valores de las variables de diseño $z$ en la Baja Escala reemplazados en $\mathrm{H}$, para calcular la descomposición en valores singulares de $\mathrm{H}$. Se recomienda tomar un paso $\Delta \mathrm{VdeC}$, con $\mathrm{VdeC}_{k}=\mathrm{VdeC}_{k-1}+\Delta \mathrm{VdeC}$, lo suficientemente grande para que el número de cálculos no sea exagerado, pero tan pequeño que evidencie (de existir) los cambios en la jerarquía dinámica, al incrementar la capacidad del proceso.

Paso 4: Normalización del Índice de Estado Impactable (IEI) y verificación del RdeO

En este paso se debe normalizar cada secuencia del $\mathrm{El}_{i}$ dividiendo por el máximo valor que alcanza el $\mathrm{IEl}_{i}$ de cada uno de los estados calculados en el paso anterior. Aparece así el IEI Normalizado ( $\left(E I_{N} i\right.$ ) que permite determinar el RdeO (jerarquía de las dinámicas).Se debe verificar que la dinámica de interés (en este ejemplo corresponde a la dinámica de producto $P$ ), sea

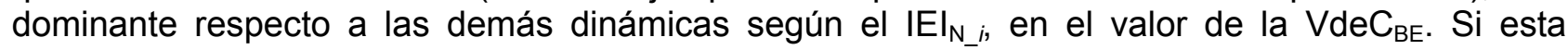
condición no se cumple, se debe regresar al Paso 1 y confirmar el valor del PdeO y de la eficiencia mínima del proceso que se desea, operando a la variable de capacidad especificada en dicho punto de operación.

Paso 5: Verificación del RdeO en todos los valores de la Variable de Capacidad (VdeC)

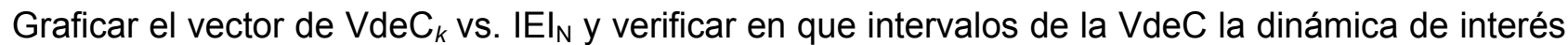
es dominante respecto a las demás dinámicas. Existen dos opciones: $i)$ El RdeO se cumple en el valor de la $\mathrm{VdeC}_{\mathrm{AE}}$, pero el $\mathrm{RdeO}$ no se cumple en valores intermedios de la VdeC. En este caso los puntos que no cumplen no se pueden utilizar como valores intermedios para el escalado (Paso 
8). ii) El RdeO no se cumple en el valor de la VdeC $\mathrm{AE}_{\mathrm{AE}}$. En este caso se debe determinar el intervalo de valores de la $\mathrm{VdeC}$ alrededor del valor de la $\mathrm{VdeC}_{\mathrm{AE}}$ que cumplen. En los demás valores de la VdeC también es posible realizar el escalado pero se debe determinar el valor del $\mid \mathrm{IEI}_{\mathrm{N}_{-} i}$ que mantendría el RdeO y proponer valores alrededor del PdeO especificado en el Paso 1 , que generen este $I E I_{N_{-} i}$. Los $I E I_{N}$ para el fermentador del ejemplo, frente a cambios en el volumen del biorreactor, se muestran en la Figura 1.

En la Figura 1 se observa que a medida que se incrementa en el volumen del biorreactor (VdeC), la dinámica del producto presenta un comportamiento dominante frente a las demás dinámicas. El comportamiento dominante de la dinámica de producto está determinado por valores del $I E I_{N}$ superiores para esta variable, frente a valores inferiores del $\mid E I_{N}$ para las demás dinámicas. Realizando en dicha gráfica un acercamiento para volúmenes inferiores a 3 litros, como se muestra en la Figura2, se observa que la dinámica de producto es dominante para volúmenes superiores a 3 litros, pero si el volumen es menor de 3 litros, la dinámica de la biomasa presenta un comportamiento dominante frente a las demás dinámicas, lo cual concuerda con el comportamiento del proceso real. Por tanto, el RdeO por debajo de 3 litros no corresponde al especificado en el Paso 1.

\section{Paso 6: Determinación del valor crítico de la Variable de Capacidad (VdeC*)}

Determinar el valor crítico de la Variable de Capacidad $\left(\mathrm{VdeC}^{*}\right)$ como el valor a partir del cual se cumpla que el mayor $\mathrm{IEI}_{\mathrm{N}_{-} i}$ corresponde al estado asociado con la dinámica de interés (lo que implica respetar el $\mathrm{RdeO}$ en su primera variable), y verificar que la eficiencia en ese $\mathrm{VdeC}{ }^{*}$ esté de acuerdo con la especificada en el Paso 1. Si esto último no se cumple, verificar que las condiciones de entrada en $\mathrm{VdeC}_{\mathrm{BE}}$ (Paso 1) son correctas y dan la eficiencia requerida. Si no es así, determinar un nuevo valor para el $\mathrm{PdeO}$ en $\mathrm{VdeC}_{\mathrm{BE}}$ (que sea superior al dado inicialmente).

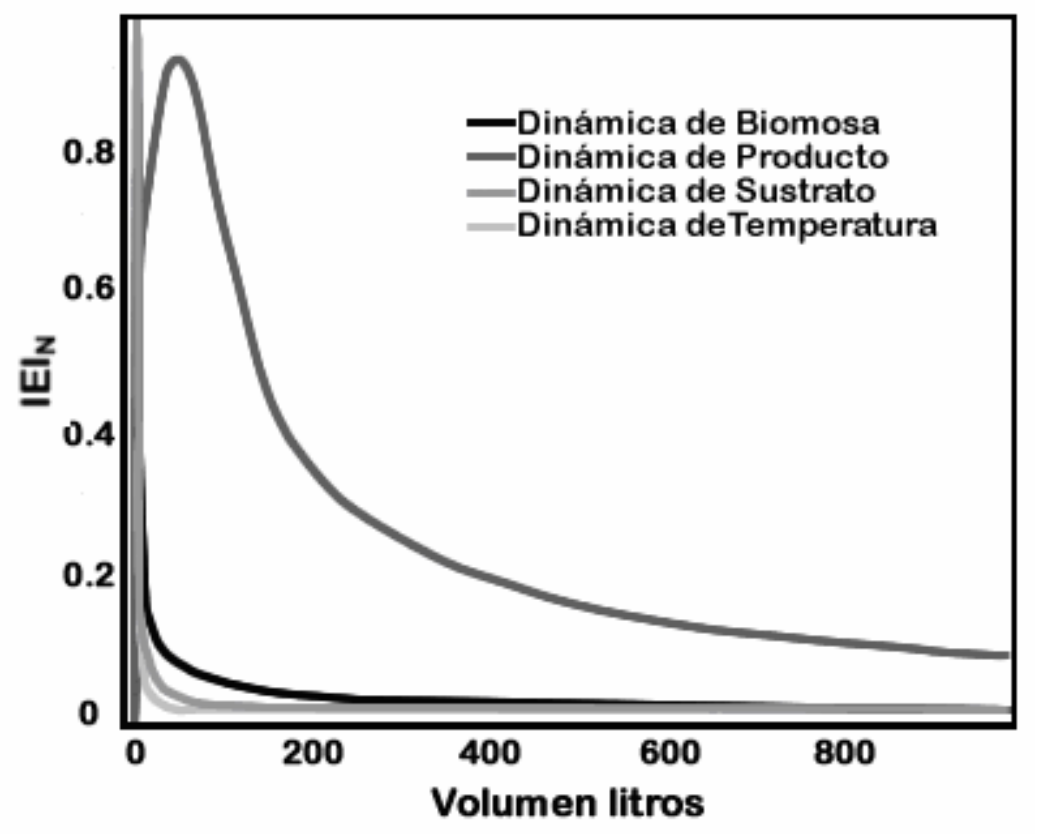

Fig.1: RdeO mediante los $I E I_{N}$ para los estados de X, S, P y T del fermentador.

En la Figura 2 se muestra valores bajos de la $V d e C$ frente al $I E I_{N}$ que permite determinar el índice de estado impactable de cada uno de los estados con las entradas como un todo. Como se ve, para el caso del fermentador del ejemplo, la dominancia de la dinámica de producto permite realizar escalado a partir de un volumen de 3 litros, en el cual se mantiene el RdeO hasta un volumen de 1000 litros. Por lo tanto en este caso el VdeC ${ }^{*}=3 \mathrm{I}$. Las dinámicas relevantes del proceso corresponden a biomasa y producto, mientras que las dinámicas de temperatura y sustrato presentan un comportamiento secundario en el sistema. Estos valores del $I E I_{N}$ fueron 
calculados con el PdeO que corresponde a la Baja Escala. En la Figura 3 se presenta el estado estacionario de la concentración de producto frente al incremento en el volumen del biorreactor, calculado con condiciones de la Baja Escala (sin ajustes de escala).

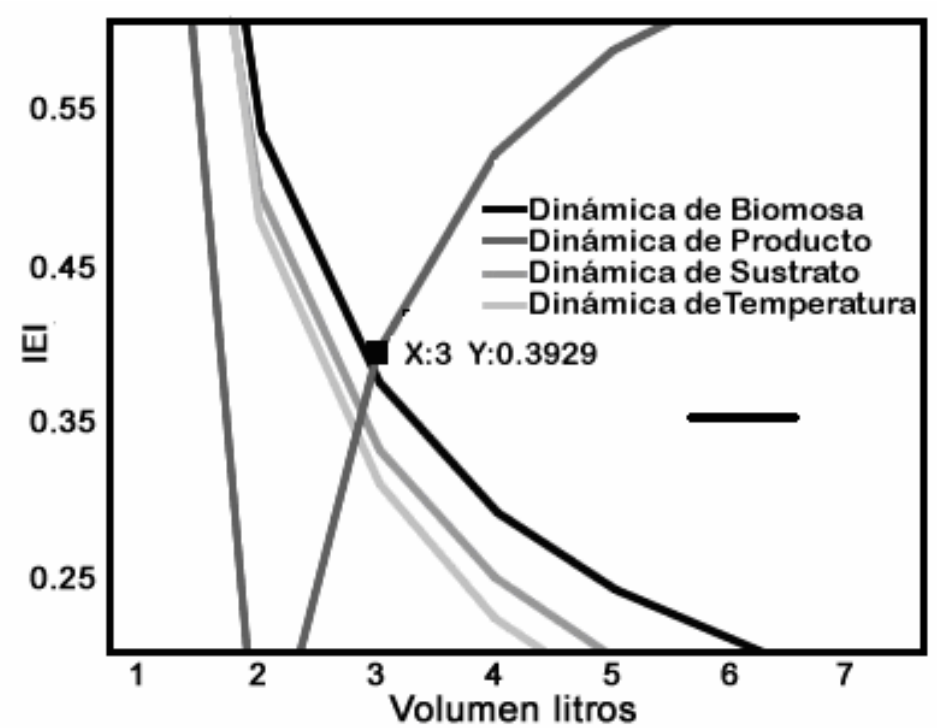

Fig.2: VdeC* según el RdeO para los estados.

Como se observa en la Figura 3, usando las condiciones de la baja escala como constantes a cualquier VdeC, para volúmenes superiores a 500 litros no se cumple con la concentración de producto esperada. Por consiguiente, con la proyección de las condiciones de operación en la escala baja, no es posible realizar un escalado de proceso para volúmenes superiores a 500 litros que cumpla con la eficiencia mínima del proceso especificada en el Paso 1. Para evitar ese error se debe realizar el escalado de forma adecuada, que como se verá más adelante, se cumple siguiendo el procedimiento propuesto en este trabajo.

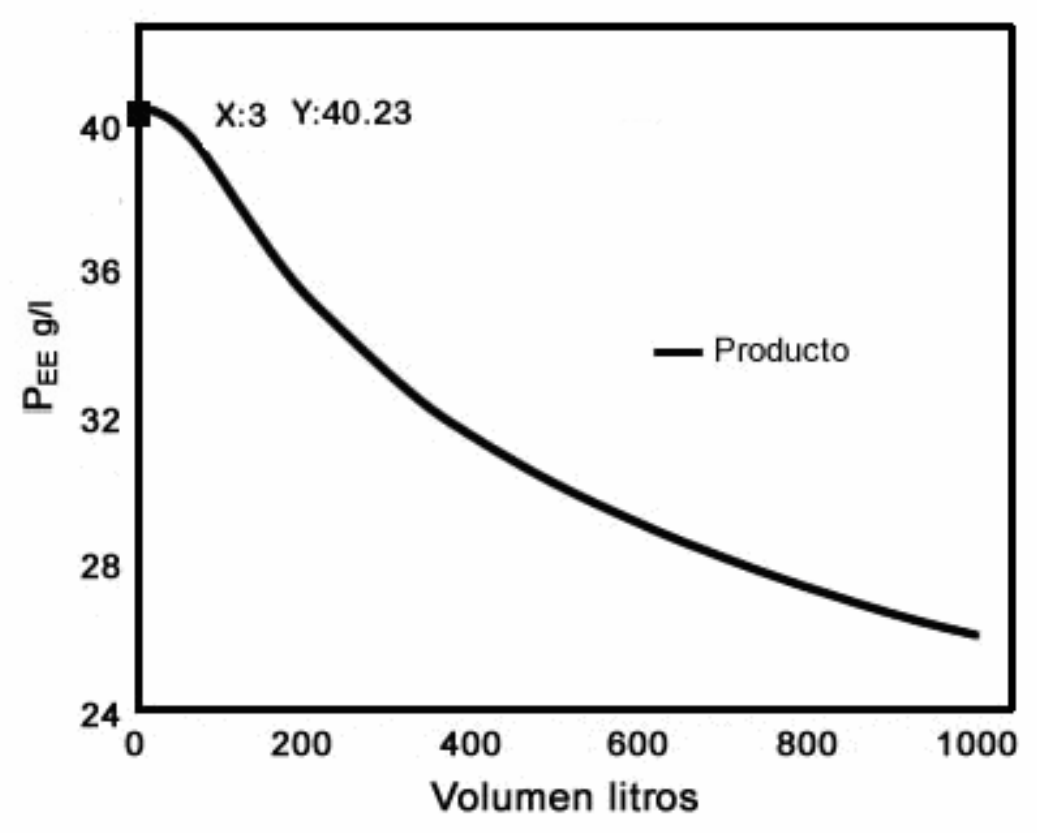

Fig.3: Estado estacionario de la concentración del producto.

Paso 7: Determinar una ecuación que permita calcular el IEl real

De acuerdo con la forma de la curva $\mathrm{VdeC}_{k} \mathrm{vs}$. $\mid \mathrm{IEI}_{\mathrm{N} \_n}$, obtenido en el Paso 6, indicando el subíndice $n$ la dinámica de interés, seleccionar un número de puntos para $\left(\mathrm{Vde}_{k}\right)$ que permitan la mejor representación de toda esa curva. Calcular en cada $\mathrm{VdeC}_{k}$ seleccionado el $\mathrm{IE} \mathrm{I}_{\mathbb{N} \_n}$ con 
condiciones de entrada del proceso reportadas o experimentales para ese $\mathrm{VdeC}_{k}$ particular. Hallar una ecuación que ajuste bien las parejas $\left(\mathrm{VdeC}_{k}, \mathrm{IEI}_{\mathrm{N}_{-} n}\right)$, para calcular $\mathrm{IEI}_{\mathrm{N}_{\_} n}=f\left(\mathrm{VdeC}_{k}\right)$. $E \mathrm{IIEI}_{\mathrm{N} \_n}$ así calculado se denominará IEI $I_{N \_n \_R e a l}$. En este caso se tomaron tres parejas de datos, puesto que la forma continua de la curva permite una buena aproximación con sólo tres puntos. La mejor ecuación de ajuste de la curva (ajuste a los puntos del 99.68\%), corresponde para el fermentador del ejemplo a:

$\mathrm{IEI}_{\mathrm{N} \mathrm{neal}}=\left(\mathrm{VdeC}_{\mathrm{F}}\right)^{0.01} \times \mathrm{IEI}_{\mathrm{N} \mathrm{n}}$

En la que IEI $I_{N \_n}$ Real es el IEI al volumen final o variable de capacidad a la que se quiere escalar $\left(V \mathrm{VeC}_{\mathrm{F}}\right), \mathrm{IEI}_{\mathrm{N} \_n}$ es el IEI al volumen final que se quiere escalar pero proyectado con las condiciones de operación en el volumen crítico (Baja Escala). De la curva correspondiente a esta ecuación se obtiene un $I E I_{N_{-} n}$ Real $=127.89$ para un volumen de 1000 litros y un $I E I_{N_{-} n}=71.85$.

\section{Paso 8: Escalar con valores entre baja escala y alta escala}

Iniciar en el valor de la $\mathrm{VdeC}_{\mathrm{BE}} \mathrm{y}$ tomando entre dos y tres valores de la $\mathrm{VdeC}$ intermedios entre $\mathrm{VdeC}_{\mathrm{BE}} \mathrm{y} \mathrm{VdeC}_{\mathrm{AE}}$, escalar como se indica a continuación. Tomar sucesivamente cada nuevo valor de la VdeC usando las condiciones de entrada de la VdeC inmediatamente anterior, hasta llevar al valor de laVdeC $\mathrm{AE}_{\mathrm{E}}$ : i) De la descomposición en valores singulares de $\mathrm{H}$, tomar las componentes $U_{n 1}, U_{n 2}, U_{n 3}, \ldots U_{n n}$ de $U$, con $n$ el número de la fila de la variable de estado de interés. ii) De la misma descomposición, tomar los valores singulares $\mathrm{s}_{11}, \mathrm{~s}_{22}, \mathrm{~s}_{33}, \ldots \mathrm{s}_{\mathrm{nn}}$ mayores que cero de $\mathrm{S}$ y calcular las proporciones de cada $\mathrm{s}_{\mathrm{ii}}$ respecto al primero $\left(\mathrm{s}_{11}\right)$. iii) Calcular la sumatoria:

$\sum U^{2}=\sum_{i=11}^{n n} U_{n n}^{2}\left[\frac{s_{n n}}{s_{11}}\right]=U_{21}^{2}\left[\frac{s_{11}}{s_{11}}\right]+U_{22}^{2}\left[\frac{s_{22}}{s_{11}}\right]+U_{23}^{2}\left[\frac{s_{33}}{s_{11}}\right]+\ldots+U_{n n}^{2}\left[\frac{s_{n n}}{s_{11}}\right]$

iv) Con el valor de la VdeC actual y la ecuación de la curva del IEI $I_{N \_n \_ \text {Real, }}$ calcular $\mid E I_{N \_n \_R e a l . V}$ ) Despejar $\mathrm{s}^{2}{ }_{11 \text {,estimado }}$ de:

$$
I E I_{n_{-} \text {real }}=\sqrt{s_{11, \text { estimado }}^{2} \sum U^{2}}
$$

Donde $n \_r e a l$ en el subíndice del IEI se refiere al Índice de Estado Impactable real para la dinámica $n$ en la alta escala.

vi) Usando las proporciones entres $s_{22}, s_{33} \ldots s_{n n} y s_{11}$, estimar nuevos $s_{i i}$ (en adelante denominados $\mathrm{s}_{\mathrm{ii}, \text { estimados }}$ ) y con ellos estimar la nueva matrizS.vii) Determinar una nueva $\mathbf{H}=\mathbf{U S e V}^{\top}$ con $\mathbf{U}$ y $\mathrm{V}$ calculadas para el valor de la $\mathrm{VdeC}$ inmediatamente anterior, con Se la matriz de valores singulares estimados. viii) Leer los valores de las $h_{i j}$ componentes de $H$, que contienen información de las condiciones de entrada del proceso a la nueva escala. ix) Igualar los valores de los $h_{i j}$ con sus ecuaciones expresadas a partir de las matrices $A, B$ y $C$ de la linealización y calcular las condiciones de entrada para la VdeC actual.

\section{Paso 9: Verificación del escalado y resultados para la alta escala}

Con las condiciones de entrada para la $\mathrm{VdeC}_{\mathrm{AE}}$, se resuelve el modelo para estimar la eficiencia del proceso en la Alta Escala y con el vector IEI a $\mathrm{VdeC}_{\mathrm{AE}}$ se verifica que el RdeO se cumpla. Si la eficiencia no se ajusta a lo esperado, se revisa el PdeO y se ajustan las condiciones de entrada hasta lograr la eficiencia deseada. En caso que alguna de las condiciones del proceso esté por fuera de los límites normales de operación, ajustarla al valor más próximo válido para esta condición (dentro del intervalo de valores permitidos para dicha variable alrededor del PdeO). Con esto se finaliza el escalado.

Al realizar este cálculo para el fermentador del ejemplo, para un volumen de 16 litros y para un 
volumen de 740.000 litros, se obtienen los resultados mostrados en la Tabla 1 , los cuales se compararon con los resultados obtenidos en la industria de destilerías en Colombia y con datos reportados en la literatura. Para cada volumen se comparan las condiciones de entrada reales con las condiciones halladas aplicando la metodología propuesta en este artículo. Para los diferentes volúmenes no se encontró diferencia en los valores obtenidos de temperatura, lo cual corresponde al comportamiento real del proceso, del cual puede decirse que autorregula la temperatura. Los errores entre los valores predichos y los entregados por el procedimiento de escalado, en todos los casos se encuentran alrededor del $10 \%$, lo cual está de acuerdo con los métodos utilizados en Ingeniería de Procesos durante las etapas de diseño. Los errores negativos corresponden a condiciones reales inferiores a las condiciones estimadas, pero que siguen estando dentro de los intervalos de valores para estas variables que hacen eficiente el proceso, lo cual se confirma con los datos de producto obtenido y guardan congruencia entre los valores de las variables, en un mismo volumen.

Tabla 1. Porcentajes de error a diferentes volúmenes entre las condiciones de entrada real y estimada con el método de escalado propuesto.

\begin{tabular}{|c|c|c|c|c|}
\hline Volumen $(\mathrm{I})$ & & Condiciones de entrada reales & Condiciones de entrada estimadas & \%error \\
\hline 16 & $\mathrm{X}_{\text {in }}$ & 1.55 & 1.40 & 9.57 \\
\hline & $\mathrm{P}_{\text {in }}$ & 3.9 & 3.51 & 9.85 \\
\hline & $\mathrm{S}_{\text {in }}$ & 220 & 196.41 & 10.72 \\
\hline & $\mathrm{T}_{\text {in }}$ & 303 & 303 & 0.00 \\
\hline & $\mathrm{Pee}$ & 12.67 & 12.90 & -1.81 \\
\hline 562500 & $\mathrm{X}_{\text {in }}$ & 64 & 68.81 & -7.51 \\
\hline & $\mathrm{P}_{\text {in }}$ & 60 & 53.51 & 10.82 \\
\hline & $\mathrm{S}_{\text {in }}$ & 470 & 470.86 & -0.18 \\
\hline & $\mathrm{T}_{\text {in }}$ & 303 & 303 & 0.00 \\
\hline & $\mathrm{Pee}$ & 53.99 & 50.26 & 6.91 \\
\hline 740000 & $\mathrm{X}_{\text {in }}$ & 64 & 63.31 & 1.08 \\
\hline & $\mathrm{P}_{\text {in }}$ & 53.1 & 49.24 & 7.21 \\
\hline & $\mathrm{S}_{\text {in }}$ & 417.7 & 433.24 & -3.72 \\
\hline & $\mathrm{T}_{\text {in }}$ & 303 & 303 & 0.00 \\
\hline & $\mathrm{Pee}$ & 49.56 & 47.25 & 4.66 \\
\hline
\end{tabular}

\section{CONCLUSIONES}

La principal dificultad en el escalado por métodos tradicionales, es no mantener la jerarquía dinámica por lo tanto se definieron, el Punto de Operación (PdeO) y el Régimen de Operación (RdeO), como herramientas para determinar si dicha jerarquía se mantiene o no durante el escalado de un proceso.

Se presentó una metodología para el escalado de procesos basada en el Modelo Semifísico de Base Fenomenológica (MSBF) del proceso y en la determinación del RdeO mediante la ponderación del efecto dinámico de las entradas como un todo sobre cada uno de los estados (Índice de Estado Impactable - IEI), a través de la descomposición en valores singulares (SVD) de la matriz de Hankel del modelo linealizado del proceso.

La metodología propuesta utiliza como representación del sistema la matriz de Hankel. Esta matriz contiene información del impacto dinámico de las entradas sobre las salidas. La matriz de Hankel en el dominio del tiempo, no tiene que evaluarse en un determinado valor, y a su vez contiene las dinámicas del proceso. Por lo tanto la matriz de Hankel tiene en cuenta el comportamiento dinámico. 
Se definió el valor crítico de la Variable de Capacidad (VdeC*), el cual indica un valor mínimo para la variable de capacidad del proceso, a partir del cual se puede aplicar la metodología de escalado manteniendo el RdeO especificado en el valor de la VdeC inicial y que garantiza la eficiencia del proceso.

Los resultados se compararon con los resultados obtenidos en la industria de destilerías en Colombia y con datos reportados en literatura. La metodología logra el escalado de procesos con errores inferiores al $10 \%$ en las condiciones obtenidas para la nueva escala, manteniendo las productividades deseadas. Es de resaltad que la metodología propuesta presenta resultados más ajustados a las condiciones reales a volúmenes industriales, que a volúmenes de laboratorio.

El modelo del fermentador contempla eventos de peso que lo acercan al proceso real, entre estos están las inhibiciones por especies (biomasa, sustrato y producto), inhibiciones de intervalo de operación ( $\mathrm{pH}$ y Temperatura) y efectos atribuidos a la agitación. Éste, es un modelo decantado, con información selecciona minuciosamente como la más apta para no sólo predecir el proceso, sino para describirlo y usar el modelo en el escalado de proceso.

\section{NOMENCLATURA}

\begin{tabular}{|c|c|}
\hline$\dot{Q}$ & Flujo de calor $[\mathrm{J} / \mathrm{s}]$ \\
\hline$\dot{\mathrm{Q}}_{\text {generado }}$ & Flujo de calor generado por la fermentación $[\mathrm{J} / \mathrm{s}]$ \\
\hline$\dot{\mathrm{Q}}_{\text {intercambio }}$ & Flujo de calor intercambiado con la chaqueta y/o el serpentín $[\mathrm{J} / \mathrm{s}]$ \\
\hline 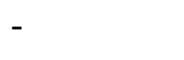 & - Sale del sistema \\
\hline+ & + Entra al sistema \\
\hline A & Constante para la correlación del Nussel \\
\hline B & Constante para la correlación del Nussel \\
\hline $\mathrm{C}$ & Constante para la correlación del Nussel \\
\hline$C_{p}$ & Calor específico. [J/kg K] \\
\hline $\mathrm{D}_{\mathrm{a}}$ & Diâmetro del agitador [m] \\
\hline$d_{\text {levadura }}$ & Diámetro de la célula $[\mu \mathrm{m}]$ \\
\hline $\mathrm{F}$ & Flujo volumétrico $\left[\mathrm{m}^{3} / \mathrm{s}\right]$ \\
\hline Gc & Factor de corrección geométrico \\
\hline $\mathrm{h}_{\mathrm{e}}$ & Coeficiente convectivo en el lado del fluido térmico $\left[\mathrm{W} / \mathrm{m}^{2} \mathrm{~K}\right]$ \\
\hline$h_{i}$ & Coeficiente convectivo en el lado del caldo $\left[\mathrm{W} / \mathrm{m}^{2} \mathrm{~K}\right]$ \\
\hline$\kappa$ & Conductividad del material del tanque \\
\hline $\mathrm{K}$ & Constante del modelo de Croughan dependiente de la célula y el reactor \\
\hline $\mathrm{K}_{\mathrm{d}}$ & Velocidad especifica de muerte $\left[\mathrm{s}^{-1}\right]$ \\
\hline $\mathrm{K}_{\mathrm{dmin}}$ & Velocidad especifica de muerte mínima $\left[\mathrm{s}^{-1}\right]$ \\
\hline $\mathrm{K}_{\mathrm{i}}$ & Constante de inhibición por sustrato $\left[\mathrm{m}^{3} / \mathrm{kg}\right]$ \\
\hline $\mathrm{K}_{\mathrm{m}}$ & Constante de mantenimiento de la célula [kg sustrato/kg célula·s] \\
\hline $\mathrm{K}_{\mathrm{s}}$ & Constante de saturación de la célula $\left[\mathrm{kg} / \mathrm{m}^{3}\right]$ \\
\hline L & Longitud característica [m] \\
\hline $\mathrm{m}$ & Constante de inhibición asociado a la biomasa \\
\hline $\mathrm{N}$ & Velocidad de agitación $\left[\mathrm{s}^{-1}\right]$ \\
\hline $\mathrm{n}$ & Constante de inhibición asociado al producto \\
\hline $\mathrm{Np}$ & Número de potencia \\
\hline$P$ & Concentración de producto $\left[\mathrm{kg} / \mathrm{m}^{3}\right]$ \\
\hline
\end{tabular}




\begin{tabular}{|c|c|}
\hline $\mathrm{pH}$ & $\mathrm{pH}$ \\
\hline $\mathrm{pH}_{\text {máx }}$ & pH máximo en el que la célula vive \\
\hline $\mathrm{pH}_{\min }$ & pH mínimo en el que la célula vive \\
\hline $\mathrm{pH}_{\mathrm{opt}}$ & pH óptimo para el crecimiento de la célula \\
\hline$P_{\text {máx }}$ & Concentración de producto máximo en el que la levadura crece $\left[\mathrm{kg} / \mathrm{m}^{3}\right]$ \\
\hline Pot & Potencia entregada por el agitador al caldo $[\mathrm{J} / \mathrm{s}][\mathrm{W}]$ \\
\hline $\operatorname{Pr}$ & Número de Prandtl \\
\hline $\operatorname{Re}$ & Número de Reynold \\
\hline$r_{p}$ & Velocidad de formación de producto $\left[\mathrm{kg} / \mathrm{m}^{3} \mathrm{~s}\right]$ \\
\hline$r_{s}$ & Velocidad de consumo de sustrato $\left[\mathrm{kg} / \mathrm{m}^{3} \mathrm{~s}\right]$ \\
\hline$r_{x}$ & Velocidad de formación de biomasa $\left[\mathrm{kg} / \mathrm{m}^{3} \mathrm{~s}\right]$ \\
\hline S & Concentración de sustrato $\left[\mathrm{kg} / \mathrm{m}^{3}\right]$ \\
\hline $\mathrm{t}$ & Tiempo de fermentación [s] \\
\hline $\mathrm{T}$ & Temperatura $[\mathrm{K}]$ \\
\hline $\mathrm{T}_{\text {máx }}$ & Temperatura máximo en el que la célula vive $[\mathrm{K}]$ \\
\hline $\mathrm{T}_{\min }$ & Temperatura mínima en el que la célula vive $[\mathrm{K}]$ \\
\hline $\mathrm{T}_{\text {opt }}$ & Temperatura óptima para el crecimiento de la célula $[\mathrm{K}]$ \\
\hline $\mathrm{T}_{\text {ref }}$ & Temperatura de referencia $[\mathrm{K}]$ \\
\hline Ug & Coeficiente global de transferencia de calor $\left[\mathrm{W} / \mathrm{m}^{2} \mathrm{~K}\right]$ \\
\hline $\mathrm{V}$ & Volumen del caldo fermentativo $\left[\mathrm{m}^{3}\right]$ \\
\hline $\mathrm{V}_{\mathrm{T}}$ & Volumen del tanque $\left[\mathrm{m}^{3}\right]$ \\
\hline$x$ & Concentración de biomasa $\left[\mathrm{kg} / \mathrm{m}^{3}\right]$ \\
\hline $\mathrm{X}_{\text {máx }}$ & Concentración de biomasa máxima en el que la levadura crece $\left[\mathrm{kg} / \mathrm{m}^{3}\right]$ \\
\hline $\mathrm{Y}_{\Delta}$ & Rendimiento de calor por sustrato consumido [kg célula/J] \\
\hline$Y_{\mathrm{p} / \mathrm{s}}$ & Rendimiento de producto por sustrato consumido [kg Producto/kg sustrato] \\
\hline $\mathrm{Y}_{\mathrm{x} / \mathrm{s}}$ & Rendimiento de biomasa por sustrato consumido [kg célula/kg Sustrato] \\
\hline$\alpha$ & Constante de producción asociada con el crecimiento \\
\hline$\beta$ & Constante de producción asociada con el mantenimiento $\left[\mathrm{s}^{-1}\right]$ \\
\hline$\varepsilon_{\mathrm{T}}$ & Energía disipada en el volumen del caldo $\left[\mathrm{m}^{2} / \mathrm{seg}^{2}\right][\mathrm{J} / \mathrm{kg} \mathrm{s}]$ \\
\hline$\lambda$ & Diámetro de los torbellinos tipo "eddy" $[\mu \mathrm{m}]$ \\
\hline$\mu$ & Velocidad específica de crecimiento $\left[\mathrm{s}^{-1}\right]$ \\
\hline$\mu_{\max }$ & Velocidad específica máxima de crecimiento $\left[\mathrm{s}^{-1}\right]$ \\
\hline$\mu_{\mathrm{opt}}$ & Velocidad específica óptima de crecimiento \\
\hline$\mu_{R}$ & Relación de viscosidades \\
\hline$v$ & Viscosidad cinemática $\left[\mathrm{m}^{2} / \mathrm{s}\right]$ \\
\hline$\rho$ & Densidad $\left[\mathrm{kg} / \mathrm{m}^{3}\right]$ \\
\hline
\end{tabular}

\section{AGRADECIMIENTOS}

Los autores expresan sus agradecimientos a Colciencias y a La Universidad Nacional de Colombia, Sede Medellín por la financiación de este proyecto. También se expresa un sincero agradecimiento al Tecnológico Universitario de Berlín (TU Berlín), por la estancia de investigación realizada por la primera autora en ese Instituto. 


\section{REFERENCIAS}

Abril, D., A. Abril, Ethanol from lignocellulosic biomass, Ciencia e investigación agraria: 36(2), 177190, (2009).

Álvarez, L. A, Metodología para el diseño de control total en planta, Tesis de Maestría, Universidad Nacional de Colombia, Medellín, Colombia (2008).

Álvarez, H., R. Lamanna, P. Vega, y S.Revollar, Metodología para la obtención de modelos semifísicos de base fenomenológica aplicada a una sulfitadora de jugo de caña de azúcar, Revista Iberoamericana de automática e informática industrial: 6(3), 10 -20, (2009).

Araya, M., J. Arrieta, J. Pérez-Correa, L. Biegler, H. Jorquera, Fast and reliable calibration of solid substrate fermentation kinetic models using advanced non-linear programming techniques, Electronic Journal of Biotechnology: 10(1), (2007).

Bastin G. y Dochain D,On-Line Estimation and Adaptive Control of Bioreactors, $1^{\text {a }}$ edición, Elsevier, New York, Estados Unidos(1990).

Bisio, A. y R. Kabel, Scale-up of chemical processes, $1^{\text {a }}$ edición, John Wiley and Sons, Estados Unidos, (1985).

Botero, H. y H. Álvarez, Una Revisión de los métodos más frecuentes para la estimación del estado en procesos químicos, Revista Dyna: 76(158), 135-146 (2009).

Botero, H. y H. Alvarez, Nonlinear state and parameters estimation in chemical processes: analysis and improvementy of three estimation structures applied to a CSTR, International Journal of Chemical Reactor Engineering: 9(A6), 1-12, (2011).

Calderón-Santoyo, M., P. Chalier, D. Chevalier-Lucia, C. Ghommidh, J. Ragazzo-Sanchez, Identification of Saccharomyces cerevisiae strains for alcoholic fermentation by discriminant factorial analysis on electronic nose signals, Electronic Journal of Biotechnology: 13(4), (2010).

Casablancas, F. G. y J.Santin,Ingeniería Bioquímica, $1^{a}$ edición, Síntesis S.A., Madrid, España (1998).

Ferreyra, M., M.C. Schvab, L. Gerard, L.M. Zapata, C.V. Davies, R.A. Hours, Fermentación alcohólica de jugo de naranja con S. cerevisiae, Ciencia, docencia y tecnología: 39, 143-158 (2009).

Johnstone, R. y M.Thring,Pilot plants, Models, and Scale-Up Methods in Chemical Engineering, Mc Graw Hill, New York, Estados Unidos (1957).

Kailath, T.,Linear Systems, $1^{\text {a }}$ edición, Pretince Hall, Estados Unidos (1980).

Kalman, R., On the general theory of control systems, First IFAC Congress, Moscow, Rusia (1960).

Kossen, N.,Scale-Up, Advances in Bioprocesses engineering: 53-65 (1996).

Lemus, Z., A. Rodríguez, M. Vistel, A. Chong, Escalado por modelación en la producción farmacéutica, Revista Cubana de Farmacia: 32(1), 7-12 (1998).

Moore, C.B., Principal component analysis in linear systems: controllability, observability and model reduction, IEEE Automatic Control: 26(1), 17-32 (1981).

Roeva, O., T. Pencheva, S. Tzonkov, M. Arndt, B. Hitzmann, S. Kleist, G. Miksch, K. Friehs, E. Flaschel, Multiple model approach to modelling of Escherichia coli fed-batch cultivation extracellular production of bacterial phytase, Electronic Journal of Biotechnology: 10(4), (2007). 
Selman, J.R., Dimensional Analysis and Scale-Up of Electrochemical Reactors, Aiche Symposium Series. Tutorial Lectures in Electrochemical Engineering and Technology II: 79(229), 101109(1983).

Wal, M. y B. Jager, A review of methods for input/output selection, Automatica: 37(4), 487-510 (2001). 